\title{
EFFICIENCY ANALYSIS OF INTEGRATED PUBLIC HOSPITAL NETWORKS IN OUTPATIENT INTERNAL MEDICINE
}

Ortiz Barrios, Miguel Angel; Escorcia Caballero, Juan P; Sánchez Sánchez, Fabián; De Felice, Fabio; Petrillo, Antonella

\begin{abstract}
Healthcare systems are evolving towards a complex network of interconnected services due to the increasing costs and the increasing expectations for high service levels. It is evidenced in the literature the importance of implementing management techniques and sophisticated methods to improve the efficiency of healthcare systems, especially in emerging economies. This paper proposes an integrated collaboration model between two public hospitals to reach the reduction of weighted average lead time in outpatient internal medicine department. A strategic framework based on value stream mapping and collaborative practices has been developed in real case study settled in Colombia.
\end{abstract}

\section{Keyword}

Efficiency, Public healthcare, Value stream mapping, Collaborative practices, DSS 